\title{
On-demand thermoelectric generation of equal-spin Cooper pairs
}

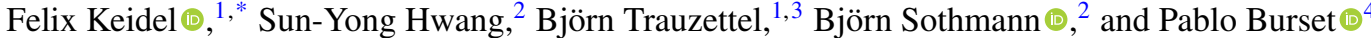 \\ ${ }^{1}$ Institute for Theoretical Physics and Astrophysics, University of Würzburg, D-97074 Würzburg, Germany \\ ${ }^{2}$ Theoretische Physik, Universität Duisburg-Essen and CENIDE, D-47048 Duisburg, Germany \\ ${ }^{3}$ Würzburg-Dresden Cluster of Excellence ct.qmat, Germany \\ ${ }^{4}$ Department of Applied Physics, Aalto University, 00076 Aalto, Finland
}

(Received 1 July 2019; accepted 13 March 2020; published 28 April 2020)

\begin{abstract}
Superconducting spintronics is based on the creation of spin-triplet Cooper pairs in ferromagnetsuperconductor (F-S) hybrid junctions. Previous proposals to manipulate spin-polarized supercurrents on demand typically require the ability to carefully control magnetic materials. We, instead, propose a quantum heat engine that generates equal-spin Cooper pairs and drives supercurrents on demand without manipulating magnetic components. We consider a S-F-S junction, connecting two leads at different temperatures, on top of the helical edge of a two-dimensional topological insulator. Heat and charge currents generated by the thermal bias are caused by different transport processes, where electron cotunneling is responsible for the heat flow to the cold lead and, strikingly, only crossed Andreev reflections contribute to the charge current. Such a purely nonlocal Andreev thermoelectric effect injects spin-polarized Cooper pairs at the superconductors, generating a supercurrent that can be switched on-off by tuning their relative phase. We further demonstrate that signatures of spin-triplet pairing are facilitated by rather low fluctuations of the thermoelectric current for temperature gradients smaller than the superconducting gap.
\end{abstract}

DOI: 10.1103/PhysRevResearch.2.022019

Introduction. The field of superconducting spintronics has emerged since the creation of spin-triplet Cooper pairs in experiments [1-3]. The development of spintronics had already benefited from the use of superconducting materials, resulting in longer spin lifetimes and energy-efficient components [4,5]. Now, triplet supercurrents formed by spin-polarized Cooper pairs add the possibility of transporting a net spin component at zero resistance and thus pave the way for spintronic devices that are less liable to overheat [6-16]. The key challenge in the field is the nonequilibrium and on-demand generation of equal-spin Cooper pairs in a viable fashion [17-21], desirably avoiding the complicated manipulation of magnetic components.

In this Rapid Communication, we propose a thermoelectric engine that creates spin-polarized Cooper pairs driving a supercurrent on demand from a temperature gradient. We consider a superconductor-ferromagnetic-insulatorsuperconductor (S-F-S) junction on top of the helical edge state of a quantum spin Hall insulator (QSHI) [22-28] connecting hot and cold baths; cf. Fig. 1(a). Only two microscopic transport processes couple the baths: quantum tunneling of electrons, known as electron cotunneling (EC) and crossed Andreev reflection (CAR). The QSHI edge states comprise one-dimensional Dirac fermions characterized by spin-

\footnotetext{
*felix.keidel@physik.uni-wuerzburg.de

Published by the American Physical Society under the terms of the Creative Commons Attribution 4.0 International license. Further distribution of this work must maintain attribution to the author(s) and the published article's title, journal citation, and DOI.
}

momentum locking [29,30]. Therefore, while EC amounts to a spin-polarized normal current, the peculiar transport properties of the helical edge states guarantee that CAR always converts electrons into holes with the same spin, creating equal-spin Cooper pairs at the superconductors [31-33]. Our key finding is that the nonlocal current becomes dominated

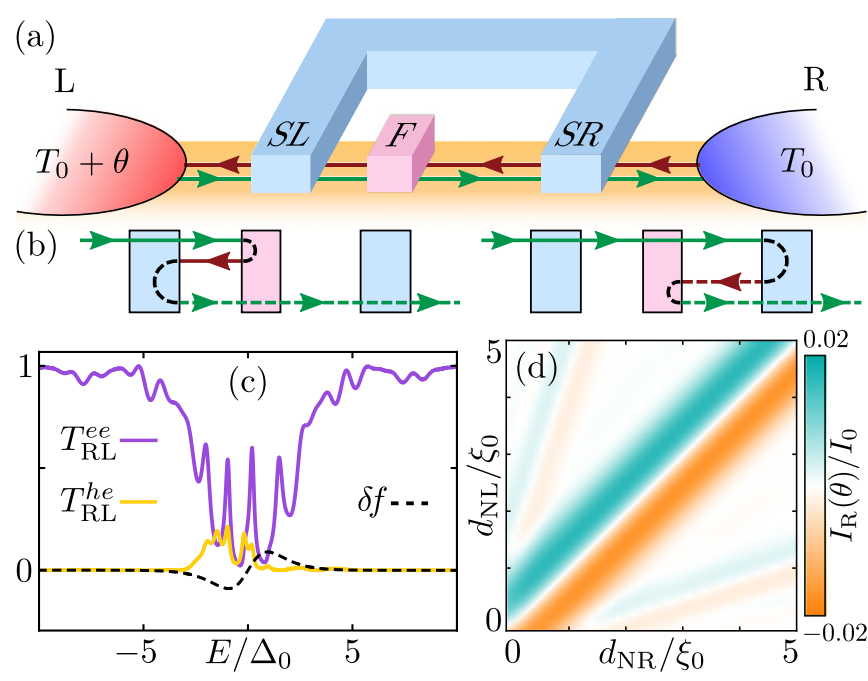

FIG. 1. Quantum heat engine generating equal-spin Cooper pairs. (a) SFS junction on the helical edge of a QSHI connecting hot (L) and cold (R) baths. (b) Lowest order contributions to equal-spin CAR. (c) Transmission probabilities for electrons $\left(T_{\mathrm{RL}}^{e e}\right)$ and holes $\left(T_{\mathrm{RL}}^{h e}\right)$ and difference in Fermi distributions $\delta f$ with $\theta=T_{c} / 4$ at $T_{0}=$ $T_{c} / 2$. (d) Unequal distance between $\mathrm{F}$ and SL $\left(d_{\mathrm{NL}}\right)$ and $\mathrm{SR}\left(d_{\mathrm{NR}}\right)$ results in an Andreev-dominated thermoelectric current $I_{\mathrm{R}}(\theta)$. 
by CAR processes, with almost complete suppression of the EC contribution. This is only possible due to a unique interference effect for CAR processes in our setup. As sketched in Fig. 1(b), CAR requires a spin-flip process at the central ferromagnet and an Andreev reflection at either the left or right superconductors. In an asymmetric junction, the different phases acquired in each path constitute interference, making CAR transmission strongly asymmetric in energy and thus creating an Andreev-dominated thermoelectric current in the right lead; cf. Figs. 1(c) and 1(d).

Harvesting waste heat by quantum thermoelectric effects has become essential in modern nanoscale devices [34]. While tackling this problem in S-F hybrid junctions can lead to potentially strong thermoelectric effects [35-44], it requires a careful control of magnetic elements and usually features a low heat-to-supercurrent conversion. In this proposal, the unique interference of CAR processes, together with the spin polarization induced by the helical edge state, creates a strong spin-polarized thermoelectric current that can be controlled by tuning the phase difference between the superconducting leads and does not rely on manipulating the ferromagnetic domain. We further demonstrate that the thermoelectric current is enhanced over its fluctuations for temperature gradients comparable to the superconducting gap, facilitating the experimental realization of our proposal by thermovoltage $[40,45]$ or thermophase measurements across the junction $[46,47]$.

Setup. The spin polarization of nonlocal transport and the absence of backscattering at the helical edge of a QSHI is of great interest for traditional spintronics. Moreover, proximityinduced superconductivity and ferromagnetism can confine the helical edge states, opening new scattering channels [31-33] that can lead to the emergence of Majorana bound states [48-51] or exotic odd-frequency superconducting pairing $[32,33,52,53]$. Given recent advances in the experimental realization of helical edge states [26-28], hybrid structures like the one sketched in Fig. 1(a) are within reach: Superconductors [54-57] have been successfully coupled to QSHIs $[58,59]$, and monolayer QSHIs provide a new promising platform to induce ferromagnetic order $[28,57]$. The observation of Majorana modes in helical hinge states of $\mathrm{Bi}(111)$ films under the influence of superconductivity and magnetic iron clusters has recently been reported in Ref. [60].

We theoretically describe the one-dimensional helical edge states of a QSHI in proximity to superconducting and ferromagnetic order by a Bogoliubov-de Gennes Hamiltonian in the Nambu basis $\Psi(x)=\left(\psi_{\uparrow}, \psi_{\downarrow}, \psi_{\downarrow}^{\dagger},-\psi_{\uparrow}^{\dagger}\right)$ of the form $\left(\hbar=v_{\mathrm{F}}=1\right)$

$$
H_{\mathrm{BdG}}=H_{0}+H_{\mathrm{S}}+H_{\mathrm{F}}
$$

with $H_{0}=\hat{p}_{x} \hat{\imath}_{3} \hat{\sigma}_{3}-\mu \hat{\tau}_{3} \hat{\sigma}_{0}$ being the Hamiltonian of the free helical edge, $H_{\mathrm{S}}=\left[\Delta(x) \cos \phi(x) \hat{\tau}_{1}+\Delta(x) \sin \phi(x) \hat{\tau}_{2}\right] \hat{\sigma}_{0}$ being the proximity-induced superconductivity, and $H_{\mathrm{F}}=$ $\hat{\tau}_{0} \mathbf{m}(x) \cdot \boldsymbol{\sigma} \equiv \hat{\tau}_{0}\left(m_{\|} \cos \lambda \hat{\sigma}_{1}+m_{\|} \sin \lambda \hat{\sigma}_{2}+m_{z} \hat{\sigma}_{3}\right)$ describing the effect of the ferromagnetic barrier. Here, $\hat{p}_{x}=-\mathrm{i} \partial_{x}$ and $\hat{\sigma}_{i}\left(\hat{\tau}_{i}\right)$ are Pauli matrices acting in spin (Nambu) space. We consider a system with two $\mathrm{S}$ regions (named SL and SR) separated by two normal regions (NL and NR) surrounding one ferromagnetic insulator $(\mathrm{F})$; their respective widths are $d_{\mathrm{X}}$

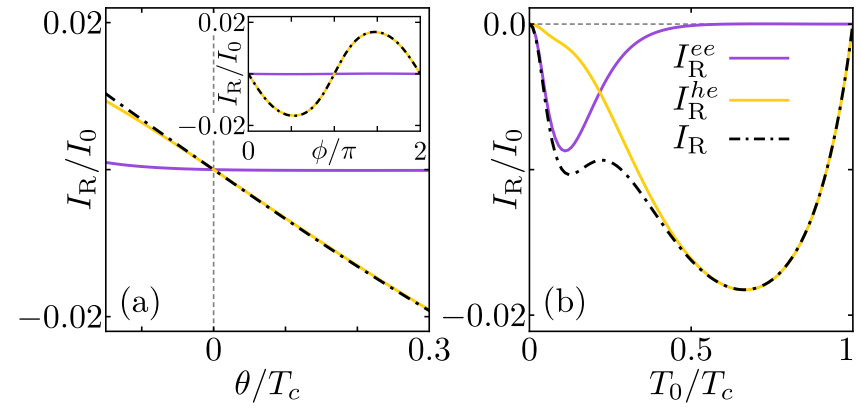

FIG. 2. Thermoelectric effect through the S-F-S junction. Total current as well as normal and Andreev contributions as a function of (a) the temperature difference (phase difference $\phi$ in the inset) and (b) the base temperature with $\theta=T_{0} / 2$ fixed. We use the parameters $d_{\mathrm{SL}}=d_{\mathrm{SR}}=\xi_{0}, d_{\mathrm{FM}}=0.6 \xi_{0}, d_{\mathrm{NL}}=0.4 \xi_{0}, d_{\mathrm{NR}}=0.9 \xi_{0}, m_{0}=1.5 \Delta_{0}$, $T_{0}=0.5 T_{c}, \phi=\pi / 2$, and $T_{c}=\Delta_{0}$ unless specified otherwise.

for $\mathrm{X} \in\{\mathrm{SL}, \mathrm{NL}, \mathrm{F}, \mathrm{NR}, \mathrm{SR}\}$. The pair potential is assumed equal for both superconductors and constant, $\Delta(x)=\Delta_{0}$, a valid approximation as long as the Fermi wavelength in each superconductor is much smaller than the proximity-induced coherence length $\xi_{0}=1 / \Delta_{0}$. For simplicity, we take the phase of the pair potential $\phi(x)=\phi$ in SR and zero otherwise. The $\mathrm{F}$ region is modeled by constant $m_{\|}(x)=m_{0}$ within $\mathrm{F}$, and we choose $m_{z}=0$ since its effect can be absorbed in the phase difference $\phi$ between the superconductors $[33,49,61]$. Without loss of generality, the angle $\lambda$ is set to zero. Finally, we assume that all regions reside at the same chemical potential, i.e., $\mu(x)=0$ everywhere.

In the following, we consider that all leads except $\mathrm{L}$ are at the same temperature [62] $\left(T_{\mathrm{SL}}=T_{\mathrm{SR}}=T_{\mathrm{R}} \equiv T_{0}\right)$ and set $T_{\mathrm{L}}=$ $T_{0}+\theta$, introducing the temperature difference $\theta$. The electric current in the right lead after a temperature bias is applied to the left lead is given by $I_{\mathrm{R}}=I_{\mathrm{R}}^{h e}+I_{\mathrm{R}}^{e e}$, where [63]

$$
\begin{aligned}
I_{\mathrm{R}}^{h e} & =I_{0} \int_{-\infty}^{\infty} \frac{d E}{\Delta_{0}} T_{\mathrm{RL}}^{h e}(E) \delta f(E), \\
I_{\mathrm{R}}^{e e} & =-I_{0} \int_{-\infty}^{\infty} \frac{d E}{\Delta_{0}} T_{\mathrm{RL}}^{e e}(E) \delta f(E),
\end{aligned}
$$

with $T_{\mathrm{RL}}^{\text {he }}$ being the CAR probability, $T_{\mathrm{RL}}^{e e}$ being the EC probability, $I_{0}=e \Delta_{0} / h, \delta f(E)=f\left[E, 0, k_{\mathrm{B}}\left(T_{0}+\theta\right)\right]-$ $f\left(E, 0, k_{\mathrm{B}} T_{0}\right)$, and $f(E, \mu, \tau)=\{1+\exp [(E-\mu) / \tau]\}^{-1}$ being the Fermi distribution function. The probabilities are obtained by solving the scattering problem defined by the solutions of Eq. (1) in every region [32,33,49,64-69]. Similarly, the current at each superconductor $\mathrm{S}=\mathrm{SL}, \mathrm{SR}$ is given by $I_{\mathrm{S}}=\left\langle J_{\mathrm{S}}\right\rangle+\int_{0}^{d_{\mathrm{S}}} d x\left\langle S_{\mathrm{S}}\right\rangle$, where $J_{\mathrm{S}}=e\left(\psi_{\mathrm{S} \uparrow}^{\dagger} \psi_{\mathrm{S} \uparrow}-\psi_{\mathrm{S} \downarrow}^{\dagger} \psi_{\mathrm{S} \downarrow}\right)$ is the quasiparticle current operator and $S_{\mathrm{S}}=i e \Delta_{0}\left(e^{-\mathrm{i} \phi_{\mathrm{S}}} \psi_{\mathrm{S} \uparrow}^{\dagger} \psi_{\mathrm{S} \downarrow}^{\dagger}+\right.$ H.c.) the source term operator [63].

As we describe in detail below, an interference of CAR processes depicted in Fig. 1(b) leads to a particular thermoelectric effect, where the current can be completely dominated by equal-spin Andreev processes; see Fig. 2 . At the same time, the energy current is only given by the symmetric part of the transmissions; therefore, it can be dominated by EC processes. Such a decoupling of transport processes for the heat and charge currents is a special feature of this setup. 

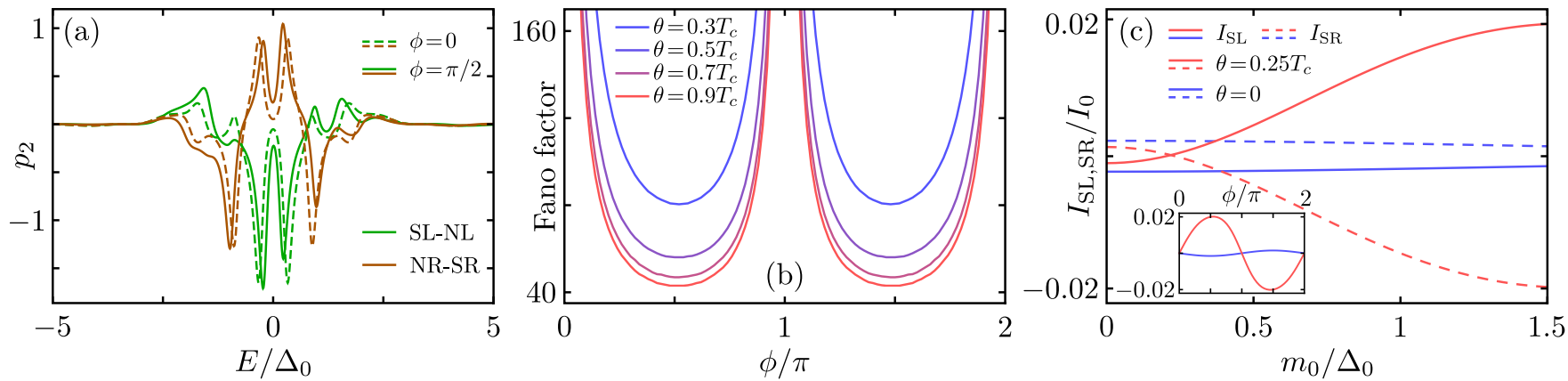

FIG. 3. (a) Cooper pair polarization $p_{2}$, computed at the SL-NL and NR-SR interfaces, as a function of the energy for fixed phase difference $\phi=\pi / 2$ ( $\phi=0$ for dashed lines). (b) Fano factor for selected temperature differences. (c) Supercurrent in SL (solid lines) and SR (dashed lines) as a function of the magnetic field strength (inset: phase difference), calculated at equilibrium (blue) and for a thermal bias $\theta=T_{c} / 4$ (red). Rest of parameters are the same as in Fig. 2.

Generation of equal-spin Cooper pairs. Helicity determines that particles arriving to the right lead have the same spin polarization as the injected particles on the left lead. While this does not restrict the quantum tunneling of electrons through the junction (EC), CAR processes are only possible if injected electrons and transmitted holes have the same spin [31-33]. By breaking time-reversal symmetry, the $F$ region facilitates equal-spin CAR processes. As sketched in Fig. 1(b), incident electrons can be transmitted as holes through the junction if at least one spin-flip process takes place at the $F$ region and one Andreev reflection occurs at either superconductor. Crucially, scattering events involving an Andreev reflection at the right superconductor will acquire an extra phase $\phi$ and a phase shift $d_{\mathrm{NR}} E$ compared to the ones where the reflection takes place at SL, which are only shifted by $d_{\mathrm{NL}} E$ (we measure $d_{\mathrm{NL}, \mathrm{NR}}$ in units of $\xi_{0}$ ). The interference between these two processes is a unique property of CAR, not present in EC, resulting in an unusually strong asymmetry of the transmission probability with the energy; cf. Fig. 1(c).

CAR processes thus require proximity-induced equal-spin pairing [32,33], which we analyze by computing the retarded Green's function associated to Eq. (1) [19,32,33]. In the basis defined above, the anomalous part of the retarded Green's function is written as $\hat{G}_{\mathrm{eh}}^{\mathrm{R}}\left(x, x^{\prime}, E\right)=f_{0}^{\mathrm{R}}\left(x, x^{\prime}, E\right) \hat{\sigma}_{0}+$ $\mathbf{f}^{\mathrm{R}}\left(x, x^{\prime}, E\right) \cdot \hat{\sigma}$, where $f_{0}^{\mathrm{R}}$ is the singlet and $\mathbf{f}^{\mathrm{R}}$ is the vector of triplet amplitudes [63]. To quantify the net spin carried by a Cooper pair, we define the polarization vector $\mathbf{p}\left(x, x^{\prime}, E\right)=$ if $\mathbf{f}^{\mathrm{R}} \times \mathbf{f}^{\mathrm{R} *}$. Note that a finite triplet amplitude is not enough to obtain a polarization. That is the case without magnetic impurity $\mathrm{F}$, where we have $\mathbf{f}^{\mathrm{R}}=\left(0,0, f_{3}^{\mathrm{R}}\right)$, but $\mathbf{p}=0$. By contrast, when time-reversal symmetry is broken by the $\mathrm{F}$ region $\left(m_{0} \neq 0\right)$, we find a finite axial polarization $\mathbf{p}(x, x, E)=$ $\left(0, p_{2}, 0\right)$, evidencing that the Cooper pairs develop a net spin.

We show in Fig. 3(a) the polarization of Cooper pairs computed at the interface between each superconductor and the inner normal regions; see Fig. 1. First, polarizations at each superconductor have different signs, indicating that Cooper pairs with opposite net spin have been transferred to each superconductor. Second, the polarization is maximum for the resonant energies of the S-F-S junction. Resonant scattering at each $\mathrm{S}-\mathrm{F}$ region always gives rise to zero-energy Majorana (quasi)bound states, with additional finite-energy Andreev states depending on the cavity's width [32,33,48,49,51]. The hybridization between the bound states at each S-F cavity is controlled by the phase difference between the superconductors $[33,61]$. This, in turn, allows for the control of the electric current through the junction.

Andreev-dominated thermoelectric effect. Given a positive temperature gradient, we find that a finite thermoelectric current, $I_{\mathrm{R}}$, is completely dominated by Andreev processes when three requirements are fulfilled (see Fig. 2): (i) The base temperature $T_{0}$ is sufficiently large, i.e., $T_{0} \gtrsim T_{c} / 2$; (ii) the junction is asymmetric, which we realize by setting $d_{\mathrm{NL}} \neq$ $d_{\mathrm{NR}}$; and (iii) the phase difference $\phi$ is not an integer multiple of $\pi$.

Under these conditions, the energy asymmetry of the CAR transmission is comparable to the energy-antisymmetric bias $\delta f$ as illustrated in Fig. 1(c), whereas the asymmetry in the EC probability occurs on a much smaller energy scale [70]. As a result, the Andreev current $I_{\mathrm{R}}^{\text {he }}$ becomes much larger than the EC current $I_{\mathrm{R}}^{e e}$ as the temperature grows. The CAR contribution is suppressed as the base temperature approaches $T_{c}$, where the induced gap vanishes. It is a good consistency check that simultaneously $I_{\mathrm{R}}^{e e} \rightarrow 0$, since without superconductivity the resonant tunneling at the $\mathrm{S}-\mathrm{F}$ regions disappears and so does the thermoelectric effect [71].

The interference effect of CAR processes is caused by an asymmetric S-F-S junction. When $d_{\mathrm{NL}}=d_{\mathrm{NR}}, I_{\mathrm{R}}^{\text {he }}$ vanishes since the two paths in Fig. 1(b) destructively interfere. In general, these two contributions acquire a different energydependent phase coming from the fact that the Andreev reflection for each path takes place at different superconductors. Consequently, a CAR-dominated thermoelectric current requires that the $\mathrm{S}-\mathrm{F}-\mathrm{S}$ junction is asymmetric and there is a finite phase difference between superconductors, as shown in Fig. 1(d). The interference effect on the CAR probability is written as [63]

$$
T_{\mathrm{RL}}^{h e}(E, \phi)=\gamma(E) \cos ^{2}\left[\phi / 2+\left(d_{\mathrm{NR}}-d_{\mathrm{NL}}\right) E\right],
$$

where $\gamma(E)$ is an even function of the energy and $\phi$ is the phase acquired by Andreev reflections at SR. Importantly, all higher order contributions are equal for both paths and even in energy [63], so they are included into the parameter $\gamma(E)$. Since only the odd part of $T_{\mathrm{RL}}^{\text {he }}$ contributes to the integration, it 
can be more conveniently expressed as

$$
T_{\mathrm{RL}}^{h e}(-E)-T_{\mathrm{RL}}^{h e}(E)=\gamma(E) \sin \left[2 E\left(d_{\mathrm{NR}}-d_{\mathrm{NL}}\right)\right] \sin \phi .
$$

The sinusoidal behavior of the current with $\phi$ is shown in the inset of Fig. 2(a), revealing the phase difference as an ideal knob to tune the thermoelectric effect. Equation (4) clearly displays two of the three conditions for the Andreevdominated thermoelectric effect. A finite electric current, $I_{\mathrm{R}}$, is obtained when the phase difference and the asymmetry result in a finite contribution to Eq. (4) that is comparable to the integration window determined by the temperature bias $\delta f\left(T_{0}, \theta\right)$. We also note that the finite thermoelectric effect indicates the simultaneous presence of both even- and oddfrequency pairing amplitudes in our setup [43].

Signatures of equal-spin Cooper pairs. An Andreevdominated thermoelectric effect stems from the injection of Cooper pairs with opposite net spin into each superconductor. The resulting current, $I_{R}$, could be measured as a voltage drop on the right lead after heating up the left one [40,72]. Our proposal is also phase tuneable so the thermoelectric power generation can be controlled by a small magnetic field [45]. Increasing the temperature gradient drives larger thermoelectric currents [see Fig. 2(a)], but also potentially larger fluctuations [73]. It is thus essential for the characterization of the proposed heat engine to identify a parameter regime where the fluctuations are the smallest with respect to the average current. That is, where the Fano factor $F=S_{\mathrm{RR}} /\left|2 e I_{\mathrm{R}}\right|$, with $S_{\mathrm{RR}}$ the current fluctuations in the right lead, is minimal. The zero-frequency fluctuation of $I_{\mathrm{R}}$ is given by [67]

$$
\begin{aligned}
S_{\mathrm{RR}}= & e I_{0} \int_{-\infty}^{\infty} \frac{d E}{\Delta_{0}} \sum_{\alpha, \beta, \gamma, \delta, k, l} \operatorname{sgn}(\alpha) \operatorname{sgn}(\beta) \\
& \times A_{k \gamma, l \delta}(\mathrm{R} \alpha, E) A_{l \delta, k \gamma}(\mathrm{R} \beta, E) f_{k \gamma}(E)\left[1-f_{l \delta}(E)\right],
\end{aligned}
$$

with

$$
A_{k \gamma, l \delta}(i \alpha, E)=\delta_{i, k} \delta_{i, l} \delta_{\alpha, \gamma} \delta_{\alpha, \delta}-\left[s_{i k}^{\alpha \gamma}(E)\right]^{*} s_{i l}^{\alpha \delta}(E),
$$

where Greek letters label Nambu indices, with $\operatorname{sgn}(\alpha)=$ \pm 1 for $\alpha=e, h$, Latin symbols represent reservoirs $\{\mathrm{L}, \mathrm{R}, \mathrm{SL}, \mathrm{SR}\}, s_{i k}^{\alpha \gamma}$ denotes the amplitude for a particle of type $\gamma$ in reservoir $k$ to be scattered into reservoir $i$ as a particle of type $\alpha$, and $f_{j \beta}(E)=f\left(E, \operatorname{sgn}(\beta) \mu_{j}, k_{\mathrm{B}} T_{j}\right)$ is the Fermi distribution for particles $\beta$ in reservoir $j$ [74].

For an asymmetric junction, the phase difference $\phi$ controls the thermoelectric current, see Eq. (4). The fluctuations are, however, almost independent of $\phi$, indicating that they are mostly caused by thermal noise [74]. As a result of the carrier-selective heat and charge transfer in this setup, thermal noise is caused by normal scattering processes that do not experience interference and $S_{\mathrm{RR}}$ increases steadily with the temperature bias $\theta$. By contrast, the Andreev-dominated current increases rapidly for $\theta<T_{c}$ and appears to saturate for higher bias. Importantly, for experimentally relevant values $[40,72]$, when the current is maximum, the Fano factor becomes minimum [see Fig. 3(b)], thus demonstrating that the current is enhanced over its fluctuations. Note that an Andreev-dominated current requires $T_{0} \gtrsim T_{c} / 2$, resulting in rather large Fano factors [63]. Recently, the electronic noise due to temperature differences in mesoscopic conductors, different than thermal or shot noise, was measured and proposed as an accurate temperature probe [75].

Finally, the injection of Cooper pairs with opposite net spin into each superconductor produces a nonequilibrium supercurrent $I_{\mathrm{SL}}(\phi)=I_{\mathrm{SR}}(-\phi)$; see Fig. 3(c). At equilibrium $(\theta=0)$, even though the current at the normal leads vanishes, $I_{\mathrm{L}, \mathrm{R}}=0$, there is a finite, Josephson-like supercurrent at each superconductor. Interestingly, without the $\mathrm{F}$ region (i.e., with $m_{0}=0$ and no CAR), the equilibrium current is barely affected by the temperature bias. By contrast, in the presence of F, CAR processes pump equal-spin Cooper pairs into the superconductors, resulting in large nonequilibrium supercurrents. The temperature bias thus creates supercurrents with opposite sign at each superconductor that could be measured separately or after connecting them through a loop, as depicted in Fig. 1. Measuring the flux inside the loop with and without temperature gradient, one could determine a thermophase [46]. Within our estimations, for biases $\theta<T_{c}$, close to the minimum of fluctuations, the magnitude of the temperature-induced supercurrent is comparable to $I_{0}$, the zero-temperature maximum Josephson current, with a typical value of $\sim 1 \mu \mathrm{A}$.

Summary. We propose a quantum heat engine that can be electrically controlled to generate spin-polarized Cooper pairs and drive supercurrents from a temperature bias on demand. Our proposal is based on a unique transport mechanism taking place at a S-F-S junction on the helical edge of a QSHI. Nonlocal Andreev processes through the junction experience an interference effect between the contributions from each superconductor. This interference is not present for normal processes, resulting in carrier-selective heat and charge currents where normal processes transfer heat and Andreev processes transfer charge. Because of the strong spin-orbit coupling at the helical edge state, the thermoelectric current is completely dominated by equal-spin Andreev processes. We discussed how the proposed spin-triplet thermoelectric effect could be measured as a thermophase appearing between the superconductors. The measurement is further facilitated by the low fluctuations of the spin-polarized nonlocal current.

Acknowledgments. The authors are grateful to $\mathrm{M}$. Moskalets for valuable discussions. We acknowledge support from the DFG (SPP 1666 and SFB 1170, Project Identification No. 258499086), the Cluster of Excellence EXC 2147 (Project Identification No. 39085490), the Ministry of Innovation NRW via the "Programm zur Förderung der Rückkehr des hochqualifizierten Forschungsnachwuchses aus dem Ausland," the Horizon 2020 research and innovation programme under the Marie Skłodowska-Curie Grant No. 743884, and the Academy of Finland (Project No. 312299).
[1] M. Eschrig, Spin-polarized supercurrents for spintronics, Phys. Today 64(1), 43 (2010).
[2] J. Linder and J. W. A. Robinson, Superconducting spintronics, Nat. Phys. 11, 307 (2015). 
[3] M. Eschrig, Spin-polarized supercurrents for spintronics: A review of current progress, Rep. Prog. Phys. 78, 104501 (2015).

[4] H. Yang, S.-H. Yang, S. Takahashi, S. Maekawa, and S. S. P. Parkin, Extremely long quasiparticle spin lifetimes in superconducting aluminium using MGO tunnel spin injectors, Nat. Mater. 9, 586 (2010).

[5] F. Hübler, M. J. Wolf, D. Beckmann, and H. v. Löhneysen, Long-Range Spin-Polarized Quasiparticle Transport in Mesoscopic Al Superconductors With a Zeeman Splitting, Phys. Rev. Lett. 109, 207001 (2012).

[6] I. Sosnin, H. Cho, V. T. Petrashov, and A. F. Volkov, Superconducting Phase Coherent Electron Transport in Proximity Conical Ferromagnets, Phys. Rev. Lett. 96, 157002 (2006).

[7] R. S. Keizer, S. T. B. Goennenwein, T. M. Klapwijk, G. Miao, G. Xiao, and A. Gupta, A spin triplet supercurrent through the half-metallic ferromagnet $\mathrm{CrO} 2$, Nature (London) 439, 825 (2006).

[8] J. W. A. Robinson, J. D. S. Witt, and M. G. Blamire, Controlled injection of spin-triplet supercurrents into a strong ferromagnet, Science 329, 59 (2010).

[9] J. W. A. Robinson, G. B. Halász, A. I. Buzdin, and M. G. Blamire, Enhanced Supercurrents in Josephson Junctions Containing Nonparallel Ferromagnetic Domains, Phys. Rev. Lett. 104, 207001 (2010).

[10] T. S. Khaire, M. A. Khasawneh, W. P. Pratt, and N. O. Birge, Observation of Spin-Triplet Superconductivity in Co-Based Josephson Junctions, Phys. Rev. Lett. 104, 137002 (2010).

[11] C. Klose, T. S. Khaire, Y. Wang, W. P. Pratt, N. O. Birge, B. J. McMorran, T. P. Ginley, J. A. Borchers, B. J. Kirby, B. B. Maranville, and J. Unguris, Optimization of Spin-Triplet Supercurrent In Ferromagnetic Josephson Junctions, Phys. Rev. Lett. 108, 127002 (2012).

[12] J. W. A. Robinson, F. Chiodi, M. Egilmez, G. B. Halász, and M. G. Blamire, Supercurrent enhancement in Bloch domain Walls, Sci. Rep. 2, 699 (2012).

[13] J. W. A. Robinson, N. Banerjee, and M. G. Blamire, Triplet pair correlations and nonmonotonic supercurrent decay with $\mathrm{Cr}$ thickness in $\mathrm{Nb} / \mathrm{Cr} / \mathrm{Fe} / \mathrm{Nb}$ Josephson devices, Phys. Rev. B 89, 104505 (2014).

[14] A. Srivastava, L. A. B. Olde Olthof, A. Di Bernardo, S. Komori, M. Amado, C. Palomares-Garcia, M. Alidoust, K. Halterman, M. G. Blamire, and J. W. A. Robinson, Magnetization Control and Transfer of Spin-Polarized Cooper Pairs into a Half-Metal Manganite, Phys. Rev. Appl. 8, 044008 (2017).

[15] S. Diesch, P. Machon, M. Wolz, C. Sürgers, D. Beckmann, W. Belzig, and E. Scheer, Creation of equal-spin triplet superconductivity at the $\mathrm{Al} / \mathrm{EuS}$ interface, Nat. Commun. 9, 5248 (2018).

[16] K.-r. Jeon, C. Ciccarelli, A. J. Ferguson, H. Kurebayashi, L. F. Cohen, X. Montiel, M. Eschrig, J. W. A. Robinson, and M. G. Blamire, Enhanced spin pumping into superconductors provides evidence for superconducting pure spin currents, Nat. Mater. 17, 499 (2018).

[17] N. Banerjee, J. W. A. Robinson, and M. G. Blamire, Reversible control of spin-polarized supercurrents in ferromagnetic Josephson junctions, Nat. Commun. 5, 4771 (2014).

[18] M. E. Bathen and J. Linder, Spin Seebeck effect and thermoelectric phenomena in superconducting hybrids with magnetic textures or spin-orbit coupling, Sci. Rep. 7, 41409 (2017).
[19] D. Breunig, P. Burset, and B. Trauzettel, Creation of spin-triplet Cooper pairs in the absence of magnetic ordering, Phys. Rev. Lett. 120, 037701 (2018).

[20] J. A. Ouassou, J. W. A. Robinson, and J. Linder, Controlling spin supercurrents via nonequilibrium spin injection, Scientific Rep. 9, 12731 (2019).

[21] J. J. He, K. Hiroki, K. Hamamoto, and N. Nagaosa, Spin supercurrent in two-dimensional superconductors with Rashba spin-orbit interaction, Commun. Phys. 2, 128 (2019).

[22] M. König, S. Wiedmann, C. Brüne, A. Roth, H. Buhmann, L. W. Molenkamp, X.-L. Qi, and S.-C. Zhang, Quantum spin Hall insulator state in HgTe quantum wells, Science 318, 766 (2007).

[23] C. Brüne, A. Roth, E. G. Novik, M. Konig, H. Buhmann, E. M. Hankiewicz, W. Hanke, J. Sinova, and L. W. Molenkamp, Evidence for the ballistic intrinsic spin Hall effect in $\mathrm{HgTe}$ nanostructures, Nat. Phys. 6, 448 (2010).

[24] I. Knez, R.-R. Du, and G. Sullivan, Evidence for Helical Edge Modes in Inverted InAs/GaSb Quantum Wells, Phys. Rev. Lett. 107, 136603 (2011).

[25] C. Brüne, A. Roth, H. Buhmann, E. M. Hankiewicz, L. W. Molenkamp, J. Maciejko, X.-L. Qi, and S.-C. Zhang, Spin polarization of the quantum spin Hall edge states, Nat. Phys. 8, 485 (2012).

[26] F. Reis, G. Li, L. Dudy, M. Bauernfeind, S. Glass, W. Hanke, R. Thomale, J. Schäfer, and R. Claessen, Bismuthene on a SiC substrate: A candidate for a high-temperature quantum spin Hall material, Science 357, 287 (2017).

[27] J. Kammhuber, M. C. Cassidy, F. Pei, M. P. Nowak, A. Vuik, Ö. Gül, D. Car, S. R. Plissard, E. P. A. M. Bakkers, M. Wimmer, and L. P. Kouwenhoven, Conductance through a helical state in an indium antimonide nanowire, Nat. Commun. 8, 478 (2017).

[28] S. Wu, V. Fatemi, Q. D. Gibson, K. Watanabe, T. Taniguchi, R. J. Cava, and P. Jarillo-Herrero, Observation of the quantum spin Hall effect up to 100 Kelvin in a monolayer, Science 359, 76 (2018).

[29] C. Wu, B. A. Bernevig, and S.-c. Zhang, Helical liquid and the edge of quantum spin Hall systems, Phys. Rev. Lett. 96, 106401 (2006).

[30] C. Xu and J. E. Moore, Stability of the quantum spin Hall effect: Effects of interactions, disorder, and $\mathbb{Z}_{2}$ topology, Phys. Rev. B 73, 045322 (2006).

[31] P. Adroguer, C. Grenier, D. Carpentier, J. Cayssol, P. Degiovanni, and E. Orignac, Probing the helical edge states of a topological insulator by Cooper-pair injection, Phys. Rev. B 82, 081303(R) (2010).

[32] F. Crépin, P. Burset, and B. Trauzettel, Odd-frequency triplet superconductivity at the helical edge of a topological insulator, Phys. Rev. B 92, 100507(R) (2015).

[33] F. Keidel, P. Burset, and B. Trauzettel, Tunable hybridization of Majorana bound states at the quantum spin Hall edge, Phys. Rev. B 97, 075408 (2018).

[34] B. Roche, P. Roulleau, T. Jullien, Y. Jompol, I. Farrer, D.A. Ritchie, and D.C. Glattli, Harvesting dissipated energy with a mesoscopic ratchet, Nat. Commun. 6, 6738 (2015).

[35] P. Machon, M. Eschrig, and W. Belzig, Nonlocal Thermoelectric Effects and Nonlocal Onsager Relations in a ThreeTerminal Proximity-Coupled Superconductor-Ferromagnet Device, Phys. Rev. Lett. 110, 047002 (2013). 
[36] M. S. Kalenkov and A. D. Zaikin, Electron-hole imbalance and large thermoelectric effect in superconducting hybrids with spin-active interfaces, Phys. Rev. B 90, 134502 (2014).

[37] M. S. Kalenkov and A. D. Zaikin, Enhancement of thermoelectric effect in diffusive superconducting bilayers with magnetic interfaces, Phys. Rev. B 91, 064504 (2015).

[38] A. Ozaeta, P. Virtanen, F. S. Bergeret, and T. T. Heikkilä, Predicted Very Large Thermoelectric Effect in FerromagnetSuperconductor Junctions in the Presence of a Spin-Splitting Magnetic Field, Phys. Rev. Lett. 112, 057001 (2014).

[39] S.-Y. Hwang, R. López, and D. Sánchez, Large thermoelectric power and figure of merit in a ferromagneticquantum dot-superconducting device, Phys. Rev. B 94, 054506 (2016).

[40] S. Kolenda, M. J. Wolf, and D. Beckmann, Observation of Thermoelectric Currents in High-Field SuperconductorFerromagnet Tunnel Junctions, Phys. Rev. Lett. 116, 097001 (2016).

[41] R. Beiranvand and H. Hamzehpour, Spin-dependent thermoelectric effects in graphene-based superconductor junctions, J. Appl. Phys. 121, 063903 (2017).

[42] Z. Cao, T.-F. Fang, L. Li, and H.-G. Luo, Thermoelectricinduced unitary Cooper pair splitting efficiency, Appl. Phys. Lett. 107, 212601 (2015).

[43] S.-Y. Hwang, P. Burset, and B. Sothmann, Odd-frequency superconductivity revealed by thermopower, Phys. Rev. B 98, 161408(R) (2018).

[44] F. S. Bergeret, M. Silaev, P. Virtanen, and T. T. Heikkilä, Colloquium: Nonequilibrium effects in superconductors with a spin-splitting field, Rev. Mod. Phys. 90, 041001 (2018).

[45] C. D. Shelly, E. A. Matrozova, and V. T. Petrashov, Resolving thermoelectric "paradox" in superconductors, Sci. Adv. 2, e1501250 (2016).

[46] F. Giazotto, T. T. Heikkilä, and F. S. Bergeret, Very Large Thermophase in Ferromagnetic Josephson Junctions, Phys. Rev. Lett. 114, 067001 (2015).

[47] F. Giazotto, P. Solinas, A. Braggio, and F. S. Bergeret, Ferromagnetic-Insulator-Based Superconducting Junctions as Sensitive Electron Thermometers, Phys. Rev. Applied 4, 044016 (2015).

[48] L. Fu and C. L. Kane, Josephson current and noise at a superconductor/quantum-spin-Hall-insulator/superconductor junction, Phys. Rev. B 79, 161408(R) (2009).

[49] F. Crépin, B. Trauzettel, and F. Dolcini, Signatures of Majorana bound states in transport properties of hybrid structures based on helical liquids, Phys. Rev. B 89, 205115 (2014).

[50] F. Crépin and B. Trauzettel, Parity Measurement in Topological Josephson Junctions, Phys. Rev. Lett. 112, 077002 (2014).

[51] C. Fleckenstein, F. Keidel, B. Trauzettel, and N. Traverso Ziani, The invisible Majorana bound state at the helical edge, Eur. Phys. J. Spec. Top. 227, 1377 (2018).

[52] J. Cayao and A. M. Black-Schaffer, Odd-frequency superconducting pairing and subgap density of states at the edge of a two-dimensional topological insulator without magnetism, Phys. Rev. B 96, 155426 (2017).

[53] C. Fleckenstein, N. T. Ziani, and B. Trauzettel, Conductance signatures of odd-frequency superconductivity in quantum spin Hall systems using a quantum point contact, Phys. Rev. B 97, 134523 (2018).
[54] I. Knez, R.-R. Du, and G. Sullivan, Andreev Reflection of Helical Edge Modes in InAs/GaSb Quantum Spin Hall Insulator, Phys. Rev. Lett. 109, 186603 (2012).

[55] S. Hart, H. Ren, T. Wagner, P. Leubner, M. Mühlbauer, C. Brüne, H. Buhmann, L. W. Molenkamp, and A. Yacoby, Induced superconductivity in the quantum spin Hall edge, Nat. Phys. 10, 638 (2014).

[56] E. Sajadi, T. Palomaki, Z. Fei, W. Zhao, P. Bement, C. Olsen, S. Luescher, X. Xu, J. A. Folk, and D. H. Cobden, Gate-induced superconductivity in a monolayer topological insulator, Science 362, 922 (2018).

[57] V. Fatemi, S. Wu, Y. Cao, L. Bretheau, Q. D. Gibson, K. Watanabe, T. Taniguchi, R. J. Cava, and P. Jarillo-Herrero, Electrically tunable low-density superconductivity in a monolayer topological insulator, Science 362, 926 (2018).

[58] J. Wiedenmann, E. Bocquillon, R. S. Deacon, S. Hartinger, O. Herrmann, T. M. Klapwijk, L. Maier, C. Ames, C. Brüne, C. Gould, A. Oiwa, K. Ishibashi, S. Tarucha, H. Buhmann, and L. W. Molenkamp, $4 \pi$-periodic Josephson supercurrent in HgTe-based topological Josephson junctions, Nat. Commun. 7, 10303 (2016).

[59] E. Bocquillon, R. S. Deacon, J. Wiedenmann, P. Leubner, T. M. Klapwijk, C. Brüne, K. Ishibashi, H. Buhmann, and L. W. Molenkamp, Gapless Andreev bound states in the quantum spin Hall insulator HgTe, Nat. Nanotechnol. 12, 137 (2017).

[60] B. Jäck, Y. Xie, J. Li, S. Jeon, B. A. Bernevig, and A. Yazdani, Observation of a Majorana zero mode in a topologically protected edge channel, Science 364, 1255 (2019).

[61] J. Nilsson, A. R. Akhmerov, and C. W. J. Beenakker, Splitting of a Cooper Pair by a Pair of Majorana Bound States, Phys. Rev. Lett. 101, 120403 (2008).

[62] To account for finite temperatures in the superconductors, the gap is approximated as $\Delta(T) \simeq \Delta_{0} \tanh \left(1.74 \sqrt{T_{c} / T-1}\right)$, with $T_{c}$ being the critical temperature and $T$ being the superconductor's temperature.

[63] See Supplemental Material at http://link.aps.org/supplemental/ 10.1103/PhysRevResearch.2.022019 for which includes Refs. [67,75-81], details of the derivation of the nonlocal current and the supercurrent, an analysis of the CAR interference, the calculation of the anomalous Green's function, and more information on the current fluctuations in the right lead.

[64] G. E. Blonder, M. Tinkham, and T. M. Klapwijk, Transition from metallic to tunneling regimes in superconducting microconstrictions: Excess current, charge imbalance, and supercurrent conversion, Phys. Rev. B 25, 4515 (1982).

[65] M. Büttiker, Scattering theory of current and intensity noise correlations in conductors and wave guides, Phys. Rev. B 46, 12485 (1992).

[66] Y. Takane and H. Ebisawa, Conductance formula for mesoscopic systems with a superconducting segment, J. Phys. Soc. Japan 61, 1685 (1992).

[67] M. P. Anantram and S. Datta, Current fluctuations in mesoscopic systems with Andreev scattering, Phys. Rev. B 53, 16390 (1996).

[68] Y. V. Nazarov and Y. M. Blanter, Quantum Transport (Cambridge University Press, Cambridge, UK, 2009).

[69] P. A. Mello and N. Kumar, Quantum Transport in Mesoscopic Systems (Oxford University Press, Oxford, UK, 2004).

[70] The physical origin of the asymmetry of EC is the spin splitting of bound states, and it is thus of the order of the hybridization 
energy. By contrast, the asymmetry in CAR is the result of an interference effect. Note that the location of the extremes of $\delta f$, and thus the width of the integration window in Eq. (2), are mainly determined by the base temperature $T_{0}$.

[71] Here, we discuss the case of a ferromagnetic insulator, as opposed to another recent proposal to use the edge states of a quantum spin Hall insulator for efficient thermoelectricity, D. Gresta, M. Real, and L. Arrachea, Optimal Thermoelectricity with Quantum Spin Hall Edge States, Phys. Rev. Lett. 123, 186801 (2019).

[72] B. Karimi and J. P. Pekola, Noninvasive Thermometer Based on the Zero-Bias Anomaly of a Superconducting Junction for Ultrasensitive Calorimetry, Phys. Rev. Appl. 10, 054048 (2018).

[73] S. Kheradsoud, N. Dashti, M. Misiorny, P. P. Potts, J. Splettstoesser, and P. Samuelsson, Power, efficiency, and fluctuations in a quantum point contact as steady-state thermoelectric heat engine, Entropy 21, 777 (2019).

[74] Since we neglect quasiparticle injection in the superconductors, the sum over the reservoirs in Eq. (5) is effectively restricted to $\mathrm{L}$ and $\mathrm{R}$.
[75] O. S. Lumbroso, L. Simine, A. Nitzan, D. Segal, and O. Tal, Electronic noise due to temperature differences in atomic-scale junctions, Nature (London) 562, 240 (2018).

[76] U. Sivan and Y. Imry, Multichannel Landauer formula for thermoelectric transport with application to thermopower near the mobility edge, Phys. Rev. B 33, 551 (1986).

[77] A. Bardas and D. Averin, Peltier effect in normal-metalsuperconductor microcontacts, Phys. Rev. B 52, 12873 (1995).

[78] J. Linder and M. E. Bathen, Spin caloritronics with superconductors: Enhanced thermoelectric effects, generalized Onsager response-matrix, and thermal spin currents, Phys. Rev. B 93, 224509 (2016).

[79] P. N. Butcher, Thermal and electrical transport formalism for electronic microstructures with many terminals, J. Phys.: Condens. Matter 2, 4869 (1990).

[80] M. Sigrist and K. Ueda, Phenomenological theory of unconventional superconductivity, Rev. Mod. Phys. 63, 239 (1991).

[81] A. J. Leggett, A theoretical description of the new phases of liquid ${ }^{3} \mathrm{He}$, Rev. Mod. Phys. 47, 331 (1975). 